\title{
Soroprevalência da doença de Chagas em cardiopatas internados em um hospital de referência da região Serrana do estado do Rio de Janeiro, Brasil
}

\section{Seroprevalence of Chagas disease in cardiac patients admitted to a reference hospital in the hills region of Rio de Janeiro State, Brazil}

\author{
1 Marco Antonio Prates Nielebock \\ 2 Fernanda Mello Regazio \\ 2 Mônica Mendes \\ 2 Renan Rocha \\ 2 Leila Figueredo \\ 3 Luiz Henrique Conde Sangenis lhcsangenis@gmail.com
}

\footnotetext{
Docente do Curso de Enfermagem da Universidade Estácio de Sá, campus Nova Friburgo, Rio de Janeiro.

Discente da Universidade Estácio de Sá, campus Nova Friburgo, Rio de Janeiro.

3 Docente do Curso de Medicina do Centro Universitário de Volta Redonda - UniFOA
}

\section{Resumo}

Objetivo: o objetivo deste estudo foi estimar a soroprevalência da doença de Chagas em cardiopatas internados em um hospital de referência da região Serrana do Rio de Janeiro e identificar fatores de risco para transmissão da doença. Material e Métodos: estudo transversal, descritivo, com utilização de questionário e teste rápido para doença de Chagas. Resultados: foram estudados 209 cardiopatas, com predomínio de idosos do sexo masculino. Moradia em casas de pau a pique nas primeiras décadas de vida, conhecimento do barbeiro e o consumo de carne de caça foram fatores de risco comuns. Insuficiência cardíaca, doença arterial coronária e arritmias foram as principais causas de internação. Uma amostra se mostrou positiva para infecção por Trypanosoma cruzi (prevalência de 0,5\%). Conclusão: apesar da baixa prevalência observada, a alta frequência de fatores de risco envolvidos na transmissão da doença de Chagas, serve de alerta para a vigilância epidemiológica do Rio de Janeiro.

\section{Palavras-chave}

Doença de Chagas; transmissão; epidemiologia; sorologia.

\begin{abstract}
Objective: The aim of this study was to estimate the prevalence of Chagas disease in cardiac admitted in a reference hospital in the hills region of Rio de Janeiro State and identify risk factors for transmission. Material and Methods: cross-sectional and descriptive study, using questionnaire and rapid test for Chagas disease. Results: a total of 209 cardiac patients were studied, with a predominance of male elderly. Living in wattle and daub dwellings in the first decades of life, kissing bugs knowledge and consumption of bush meat were common risk factor. Heart failure, coronary artery disease and arrhythmias were the main causes of hospitalization. One sample was positive for Trypanosoma cruzi infection (prevalence of 0.5\%). Conclusion: despite the low prevalence noted, the high frequency of risk factors involved in the transmission of Chagas disease serves as a warning to epidemiological surveillance of Rio de Janeiro.
\end{abstract}

\section{Keywords}

Chagas disease; transmission; epidemiology; serology.

\section{Como você deve citar?}

SANGENIS, Luiz Henrique Conde et al. Soroprevalência da doença de Chagas em cardiopatas internados em um hospital de referência da região Serrana do estado do Rio de Janeiro, Brasil. Cadernos UniFOA, Volta Redonda, $\mathrm{n}$. 33, p. 131-138, abr. 2017. 
Soroprevalência da doença de Chagas em cardiopatas internados em um hospital de referência da região Serrana do estado do Rio de Janeiro, Brasil

\section{INTRODUÇÃO}

A infecção por Trypanosoma cruzi é causa importante de doença cardíaca na América Latina com índices elevados de morbidade e mortalidade (MONCAYO; SILVEIRA, 2009; WHO, 2012). Aproximadamente $30 \%$ dos pacientes com doença de Chagas (DC) apresentam algum comprometimento cardíaco (RASSI et al., 2010). Estima-se que anualmente ocorram 12.500 óbitos por complicações da doença de Chagas (DC) no mundo (WHO, 2012). O Brasil registra anualmente mais de 5000 óbitos relacionados à DC, sendo a maior parte devido a complicações cardíacas (MARTINS-MELO et al., 2012; NÓBREGA et al., 2014). Morte súbita, falência cardíaca e acidente vascular cerebral são as principais causas de óbito em portadores de DC (BIOLO et al., 2010; RASSI et al., 2010). Além disso, a cardiopatia chagásica crônica é importante causa de anos de vida perdidos em indivíduos jovens economicamente ativos, gerando um grande impacto social, econômico e na saúde pública dos países endêmicos (MONCAYO; SILVEIRA, 2009; WHO, 2012).

A prevalência da DC na América Latina vem reduzindo nos últimos anos devido a bem sucedidas campanhas de combate aos principais vetores (Triatoma infestans e Rhodnius prolixus) e políticas de controle de doadores de sangue (MONCAYO; SILVEIRA, 2009). No entanto, estima-se que ainda existam cerca de 8 milhões de portadores e 28 milhões de indivíduos sob risco de adquirir a doença (MONCAYO; SILVEIRA, 2009; WHO, 2012).

O estado do Rio de Janeiro (RJ) nunca foi considerado endêmico para DC, porém, estudos recentes identificaram a presença da transmissão autóctone em áreas rurais do estado, particularmente em municípios das regiões Serrana e Norte (LOROSA et al., 2008; SANGENIS et al., 2015a). Ciclos silvestres de T. cruzi foram identificados em localidades rurais do RJ, assim como a invasão domiciliar por triatomíneos nativos da Mata Atlântica que podem ser implicados na transmissão vetorial da DC (GONÇALVES et al., 1998; LOROSA et al., 2003, 2008; SANGENIS et al., 2015a, 2015b). Dentre os pacientes portadores de DC adquirida no RJ predominou a forma cardíaca e alguns casos evoluíram para o óbito (SANGENIS et al., 2015a).

O objetivo deste estudo foi estimar a prevalência da DC entre cardiopatas assistidos no Hospital Municipal Raul Sertã, em Nova Friburgo, unidade terciária que serve de referência médica para 12 municípios da região Serrana do RJ, assim como identificar fatores de risco envolvidos na transmissão da infecção por T. cruzi entre os indivíduos investigados.

\section{MATERIAL E MÉTODOS}

\subsection{Desenho do estudo, casuística e obtenção de amostras}

Trata-se de estudo descritivo do tipo transversal com utilização de questionário semiestruturado para identificar fatores de risco associados à aquisição da DC e inquérito sorológico da população estudada. Foram incluídos no estudo todos os pacientes com cardiopatia de qualquer natureza, maiores de 18 anos e internados no Hospital Municipal Raul Sertã, em Nova Friburgo, no período de fevereiro a outubro de 2015. Para o recrutamento foram realizadas visitas semanais ao hospital de segunda a sexta-feira, onde se priorizou as enfermarias de clínica médica, unidade coronariana e unidade de terapia intensiva. Os pacientes foram inicialmente identificados pelo corpo clínico (médicos e enfermeiros) dos diversos setores do hospital como portadores de cardiopatia e convidados a participar da pesquisa. Para facilitar a identificação e resposta sobre o conhecimento do "barbeiro", foram utilizados mostruários com fotos coloridas do inseto. Amostras de sangue foram coletadas através de punção da poupa digital para realização de teste rápido pela técnica de imunocromatografia (TR Chagas ${ }^{\circledR}$ ) 
desenvolvido pelo laboratório BioManguinhos/Fiocruz e com sensibilidade e especificidade de $98,5 \%$ e 100\% respectivamente e leitura em 20 minutos. Testes rápidos pela técnica de imunocromatografia mostraram-se adequados no diagnóstico sorológico de DC (AFONSO et al., 2012). Métodos sorológicos convencionais para DC pelas técnicas de imunofluorescência indireta e ELISA foram repetidos naqueles que apresentaram teste rápido positivo.

\subsection{Análise estatística}

Os resultados foram analisados de forma descritiva, utilizando-se frequências absolutas e porcentagens. A ferramenta utilizada para análise foi o software Epi Info na versão 3.5.2.

\subsection{Aspectos éticos}

A pesquisa foi aprovada pelo Comitê de Ética em Pesquisa em Seres Humanos do Instituto Nacional de Infectologia Evandro Chagas, Fundação Oswaldo Cruz, com parecer número 990.233. Os participantes que concordaram em participar da pesquisa foram orientados individualmente sobre 0 trabalho e assinaram o termo de consentimento livre esclarecido (TCLE).

\section{RESULTADOS}

Foram incluídos no estudo 209 pacientes com cardiopatia de qualquer etiologia com idade superior a 18 anos. Houve predomínio de indivíduos brancos e do sexo masculino. A maior parte dos pacientes era natural de municípios da região Serrana do RJ, tinha histórico de moradia em áreas rurais nas primeiras décadas de vida, baixa escolaridade e negaram ter mãe ou irmãos com DC. A moradia em casas de pau a pique e o consumo de carne de caça de animais silvestres foram hábitos comuns. Em relação ao conhecimento do vetor, $97(46,4 \%)$ referiu conhecer o barbeiro, no entanto, somente 27 $(12,9 \%)$ pacientes relataram ter visto o inseto dentro de casa (Tabela 1).

Em relação ao diagnóstico da cardiopatia que motivou a internação, a mais frequente foi a insuficiência cardíaca congestiva (ICC), seguida da doença arterial coronária (DAC), das arritmias e da insuficiência ventricular esquerda (IVE). Valvulopatias, pericardites e cardiomiopatias de outra natureza foram doenças menos frequentes. Hipertensão arterial sistêmica e diabetes mellitus foram comorbidades frequentes, $73,2 \%$ e $36,4 \%$ respectivamente. Dentre outras comorbidades relatadas, 0 acidente vascular cerebral, o alcoolismo e o tabagismo foram os agravos mais citados. Dois pacientes referiram possuir marcapasso definitivo implantado. Apenas um paciente apresentou positividade para infecção por T. cruzi no teste rápido, tratando-se de um homem de 68 anos, natural do estado de Minas Gerais, residente em Nova Friburgo e portador de ICC. Com isso, a prevalência de DC na população estudada foi de $0,5 \%$ (Tabela 2). 
Soroprevalência da doença de Chagas em cardiopatas internados em um hospital de referência da região Serrana do estado do Rio de Janeiro, Brasil

Tabela 1 - Variáveis sociodemográficas e epidemiológicas dos pacientes internados com cardiopatia (N=209).

\begin{tabular}{|c|c|c|}
\hline Variáveis & Número & Porcentagem (\%) \\
\hline \multicolumn{3}{|l|}{ Sexo } \\
\hline Feminino & 90 & 43,1 \\
\hline Masculino & 119 & 56,9 \\
\hline \multicolumn{3}{|l|}{ Idade } \\
\hline 18 a 59 anos & 70 & 33,5 \\
\hline 60 a 93 anos & 139 & 66,5 \\
\hline \multicolumn{3}{|l|}{ Cor } \\
\hline Branca & 135 & 64,6 \\
\hline Preta & 34 & 16,3 \\
\hline Parda & 40 & 19,1 \\
\hline \multicolumn{3}{|l|}{ Escolaridade } \\
\hline Analfabeto & 36 & 17,2 \\
\hline Fundamental incompleto & 109 & 52,2 \\
\hline Fundamental completo & 29 & 13,9 \\
\hline Médio & 28 & 13,4 \\
\hline Superior & 7 & 3,3 \\
\hline \multicolumn{3}{|l|}{ Naturalidade } \\
\hline RJ & 200 & 95,7 \\
\hline MG & 5 & 2,4 \\
\hline PB & 1 & 0,5 \\
\hline $\mathrm{PI}$ & 1 & 0,5 \\
\hline RS & 1 & 0,5 \\
\hline $\mathrm{SP}$ & 1 & 0,5 \\
\hline \multicolumn{3}{|l|}{ Origem } \\
\hline Rural & 159 & 76 \\
\hline Urbana & 50 & 24 \\
\hline \multicolumn{3}{|c|}{ Moradia em casa de pau a pique } \\
\hline Não & 89 & 42,6 \\
\hline Sim & 120 & 57,4 \\
\hline \multicolumn{3}{|l|}{ Conhecimento do "barbeiro" } \\
\hline Não & 112 & 53,6 \\
\hline $\operatorname{Sim}$ & 97 & 46,4 \\
\hline \multicolumn{3}{|l|}{ Viu o inseto dentro de casa? } \\
\hline Não & 182 & 87,1 \\
\hline Sim & 27 & 12,9 \\
\hline \multicolumn{3}{|c|}{ Consumiu carne de caça (mamíferos silvestres)? } \\
\hline \multirow{2}{*}{$\begin{array}{l}\text { Não } \\
\text { Sim }\end{array}$} & 48 & 23 \\
\hline & 161 & 77 \\
\hline \multicolumn{3}{|l|}{ Recebeu transfusão de sangue? } \\
\hline Não & 173 & 82,8 \\
\hline Sim & 36 & 17,2 \\
\hline
\end{tabular}


Marco Antonio Prates Nielebock / Fernanda Mello Regazio / Mônica Mendes Renan Rocha / Leila Figueredo / Luiz Henrique Conde Sangenis

Tabela 2 - Dados clínicos e sororreatividade para doença de Chagas dos pacientes internados com cardiopatia. (N=209).

\begin{tabular}{|c|c|c|}
\hline Dados clínicos & Número & Porcentagem (\%) \\
\hline \multicolumn{3}{|l|}{ Diagnóstico da cardiopatia } \\
\hline Insuficiência cardíaca congestiva & 97 & 46,4 \\
\hline Doença arterial coronária & 41 & 19,6 \\
\hline Arritmias & 40 & 19,1 \\
\hline Insuficiência ventricular esquerda & 21 & 10,0 \\
\hline Valvulopatias & 4 & 2,0 \\
\hline Pericardites & 2 & 1,0 \\
\hline Outras cardiomiopatias & 4 & 2,0 \\
\hline \multicolumn{3}{|l|}{ Tem hipertensão arterial? } \\
\hline Não & 56 & 26,8 \\
\hline Sim & 153 & 73,2 \\
\hline \multicolumn{3}{|l|}{ Tem diabetes? } \\
\hline Não & 133 & 63,6 \\
\hline $\operatorname{Sim}$ & 76 & 36,4 \\
\hline \multicolumn{3}{|l|}{ Comorbidades } \\
\hline Acidente Vascular Cerebral & 22 & 10,5 \\
\hline Alcoolismo & 17 & 8,1 \\
\hline Tabagismo & 15 & 7,2 \\
\hline Insuficiência renal crônica & 7 & 3,3 \\
\hline Anemia & 6 & 2,9 \\
\hline Doença pulmonar obstrutiva crônica & 6 & 2,9 \\
\hline Pneumonia & 5 & 2,4 \\
\hline Infecção urinária & 4 & 1,9 \\
\hline Doença de Alzheimer & 3 & 1,4 \\
\hline Depressão & 2 & 1,0 \\
\hline Derrame pleural & 2 & 1,0 \\
\hline Trombose venosa profunda & 2 & 1,0 \\
\hline Cirrose hepática & 2 & 1,0 \\
\hline Câncer de próstata & 2 & 1,0 \\
\hline Artrose & 2 & 1,0 \\
\hline Artrite reumatoide & 1 & 0,5 \\
\hline Mieloma múltiplo & 1 & 0,5 \\
\hline Síndrome do pânico & 1 & 0,5 \\
\hline Obesidade mórbida & 1 & 0,5 \\
\hline \multicolumn{3}{|l|}{ Sororreatividade para doença de Chagas } \\
\hline Negativa & 208 & 99,5 \\
\hline Positiva & 1 & 0,5 \\
\hline
\end{tabular}


Soroprevalência da doença de Chagas em cardiopatas internados em um hospital de referência da região Serrana do estado do Rio de Janeiro, Brasil

\section{DISCUSSÃo}

As características sociodemográficas e clínicas observadas nos pacientes cardiopatas estudados se assemelharam a outros estudos desenvolvidos em unidades de saúde pública no Brasil com o mesmo tipo de clientela (BOPP; BARBIERO, 2009; PEREIRA-BARRETO et al., 2013). Chamou a atenção o número expressivo de pacientes que referiram conhecer o "barbeiro". No entanto, somente 12,9\% dos indivíduos relataram ter visto o inseto dentro de casa. Na região Serrana do RJ, a invasão domiciliar por triatomíneos silvestres é um fato comum, principalmente nas áreas rurais, origem da maior parte dos pacientes deste estudo (LENT, 1942; FERREIRA et al., 1986; GONÇALVES et al., 1998; SANGENIS et al., 2015). Vários estudos documentaram a presença de Triatoma vitticeps em domicílios da região Serrana, com altas taxas de infecção por T. cruzi, sendo atraídos pelas luzes das casas à noite (GONÇALVES et al., 1998; LOROSA et al., 2003; 2008, SANGENIS et al., 2015a). Apesar de grande parte dos pacientes ter habitado casas de pau a pique, esse dado parece não ser importante na epidemiologia da DC no RJ, visto que a colonização dos domicílios por vetores nativos raramente ocorre (GONÇALVES et al., 1998; SANGENIS et al., 2015a). Outra característica de risco observada entre os pacientes deste estudo foi o hábito muito comum de consumir carne de caça de mamíferos silvestres, o que pode ser implicado na transmissão da infecção por T. cruzi (DIAS; AMATO-NETO, 2011; TOSO et al., 2011). Estudos desenvolvidos anteriormente em áreas rurais de São Paulo atribuiu a transmissão humana de T.cruzi ao contato com carcaças e ao consumo de carne de caça de mamíferos silvestres (FORATTINI et al., 1980; CARVALHO et al., 2003).

A DC é considerada uma causa importante de insuficiência cardíaca e de óbitos em diversas regiões do Brasil (BOCCHI et al., 2009; MARTIN-MELO et al., 2012). Embora a ICC tenha sido a cardiopatia mais observada no presente estudo, a infecção por $T$. cruzi não foi etiologia importante dessa complicação, visto que a prevalência de DC na população estudada ( $0,5 \%)$ foi baixa. Provavelmente os fatores desencadeantes de ICC observados neste trabalho tenham sido a hipertensão arterial e a doença coronária, que são causas mais frequentes de complicações cardíacas no Brasil (BOCCHI et al., 2009). No Brasil, as doenças cardiovasculares são a principal causa de internação entre os idosos (COSTA et al., 2000). ICC e DAC respondem pela maior parte das hospitalizações, o que também foi observado no presente estudo (BOCCHI et al., 2009; LENTSCK et al., 2015)

O único caso positivo para DC no teste rápido entre os voluntários do estudo foi um homem de 68 anos natural de Minas Gerais, do município de Itamarandiba, região do Vale do Jequitinhonha, pertencente à área endêmica original de DC no Brasil (SILVEIRA; DIAS, 2011). O paciente relatou ser de origem rural, morou em casa de pau a pique na infância, conhecia o barbeiro e frequentemente os via dentro do domicílio. Negou ter recebido transfusões de sangue e referiu ter se afastado da área rural aos 15 anos de idade, quando se mudou para o RJ, fortalecendo a hipótese de caso importado de DC. Há cinco anos vinha se queixando de dispneia progressiva aos esforços, astenia e edemas em membros inferiores. Negou histórico de HAS e de DAC. O diagnóstico de DC foi confirmado por testes sorológicos convencionais, pelos métodos de imunofluorescência indireta e ELISA que também se mostraram positivos.

Apesar das características epidemiológicas dos indivíduos naturais do RJ encontradas neste estudo ser semelhante a outras regiões endêmicas de DC do Brasil (MAGALHÃES et al., 2011; BRITO et al., 2012; ARAÚJO et al., 2015; LIMA et al., 2015), o provável baixo potencial vetorial dos triatomíneos nativos, como o $T$. vitticeps, deve ter contribuído para ausência de casos positivos entre os fluminenses (GONÇALVES et al., 1998; SANGENIS et al., 2015). Limitações relacionadas à sensibilidade e execução do teste diagnóstico utilizado também podem ter influído no resultado. Além disso, estudos transversais apresentam limitações em detectar doenças raras, obrigando a seleção de grande número de amostras. Provavelmente o número de 209 voluntários foi insuficiente para estimar a prevalência da DC dentre os cardiopatas da área de abrangência do estudo. Apesar da baixa prevalência observada, a frequência de 
fatores de risco para transmissão da DC relatados, como a visualização de vetores no intradomicílio e o hábito de consumir animais silvestres, serve de alerta à vigilância epidemiológica do RJ.

\section{AGRADECIMENTOS}

Agradecemos a Edimilson Domingos da Silva, Departamento de Reativos Imunobiológicos do Bio-Manguinhos/Fundação Oswaldo Cruz, pelo fornecimento dos testes rápidos para esta pesquisa. Este estudo foi realizado em colaboração do Laboratório de pesquisa Clínica em doença de Chagas do Instituto Nacional de Infectologia, Fundação Oswaldo Cruz, com a Universidade Estácio de Sá, Rio de Janeiro, através de projeto de extensão universitária Pesquisa e Produtividade.

\section{REFERÊNCIAS}

AFONSO, A.M.; EBELL, M.H.; TARLETON, R.L. A Systematic Review of High Quality Diagnostic Tests for Chagas Disease. PLoS Negl. Trop. Dis., v. 6, p. e1881, 2012.

ARAÚJO, A.C.; RODRIGUES, S.C.; REZENDE, A.F.S. et al. Soroprevalência de infecção humana por Trypanosoma cruzi em uma área rural do sul do Brasil. Rev. Patol. Trop., v. 44, n. 4, p. 423-431, 2015.

BIOLO, A.; RIBEIRO, A.L.; CLAUSELL, N. Chagas cardiomyopathy-where do we stand after a hundred years? Prog. Cardiovasc. Dis., v. 52, n. 4, p. 300-316, 2010.

BOCCHI, E.A.; MARCONDES-BRAGA, F.G.; AYUB-FERREIRA, S.M. et al. Sociedade Brasileira de Cardiologia. III Diretriz Brasileira de Insuficiência Cardíaca Crônica. Arq. Bras. Cardiol., v. 93, n. 1, p. 1-71, 2009. Suplemento1.

BOPP, M.; BARBIERO, S. Prevalência de síndrome metabólica em pacientes de um ambulatório do Instituto de Cardiologia do Rio Grande do Sul (RS). Arq. Bras. Cardiol., v. 93, n. 5, p. 473-477, 2009.

BRITO, C.R.N.; SAMPAIO, G.H.F.; CÂMARA, A.C.J. et al. Seroepidemiology of Trypanosoma cruzi infection in the semiarid rural zone of the State of Rio Grande do Norte, Brazil. Rev. Soc. Bras. Med. Trop., v. 45, n. 3, p. 346-352, 2012.

CARVALHO, M.E.; SILVA, R.A.; BARATA, J.M.S. et al. Soroepidemiologia da tripanossomíase americana na região do litoral sul, São Paulo. Rev. Saúde Pública, v. 37, n. 1, p. 49-58, 2003.

COSTA, M.F.F.L.; GUERRA, H.L.; BARRETO, S.M. et al. Diagnóstico da situação de saúde da população idosa brasileira: um estudo da mortalidade e das internações hospitalares públicas. Inf. Epidemiol. SUS, v. 9, n. 1, p. 23-41, 2000.

DIAS, J.C.P.; AMATO-NETO, V. Prevenção referente às modalidades alternativas de transmissão do Trypanosoma cruzi no Brasil. Rev. Soc. Bras. Med. Trop., v. 44, p. 67-72, 2011. Suplemento II.

FERREIRA, E.; SOUZA, O.S.; FONSECA-FILHO, M. et al. Nota sobre a distribuição geográfica do Triatoma vitticeps Stal, 1859 (Hemiptera, Reduvidae) no estado do Rio de Janeiro, Brasil. Rev. Brasil. Malariol. D. Trop., v. 38, p. 11-14, 1986.

FORATTINI, O.P.; SILVA, E.O.R.; BARATA, J.M.S. et al. Nota sobre caso autóctone de tripanossomíase americana no litoral sul do estado de São Paulo, Brasil. Rev. Saúde Pública, v. 14, p. 143-149, 1980. 
Soroprevalência da doença de Chagas em cardiopatas internados em um hospital de referência da região Serrana do estado do Rio de Janeiro, Brasil

GONÇALVES, T.C.M.; OLIVEIRA, E; DIAS, L.S. et al. An investigation on the ecology of Triatoma vitticeps (Stal, 1859) and its possible role in the transmission of Trypanosoma cruzi, in the locality of Triunfo, Santa Maria Madalena municipal district, state of Rio de Janeiro, Brazil. Mem. Inst. Oswaldo Cruz, v. 93, n. 6, p. 711-717, 1998.

LENT, H. Transmissores da moléstia de Chagas no Estado do Rio de Janeiro. Rev. Flum. Med., v. 6, p. 7-19, 1942.

LENTSCK, M.H.; LATORRE, R.D.O.; MATHIAS, T.A.F. Trends in hospitalization due to cardiovascular conditions sensitive to primary health care. Rev. Bras. Epidemiol., v. 18, n. 2, p. 372-384, 2015.

LIMA, M.M.; CARVALHO-COSTA, F.A.; TOMA, H.K. et al. Chagas disease and housing improvement in northeastern Brazil: a cross-sectional survey. Parasitol. Res., v. 114, n. 5, p. 1687-1692, 2015.

LOROSA, E.S.; SANTOS, C.M.; JUBERG, J. Foco da doença de Chagas em São Fidélis, no Estado do Rio de Janeiro. Rev. Soc. Bras. Med. Trop., v. 41, n. 4, p. 419-420, 2008.

LOROSA, E.S.; VALENTE, M.V.M.; CUNHA, V. et al. Foco da doença de Chagas em Arcádia, Estado do Rio de Janeiro, Brasil. Mem. Inst. Oswaldo Cruz, v. 98, n. 7, p. 885-887, 2003.

MAGALHÃES, B.M.L.; COELHO, L.I.A.R.C.; MACIEL, M.G. et al. Serological survey for Chagas disease in the rural areas of Manaus, Coari, and Tefé in the Western Brazilian Amazon. Rev. Soc. Bras. Med. Trop., v. 44, n. 6, p. 697- 702, 2011.

MARTINS-MELO, F.R.; ALENCAR, C.H.; RAMOS, A.N. et al. Epidemiology of mortality related to Chagas' disease in Brazil, 1999-2007. PLoS Negl. Trop. Dis., v. 6, p. e1508, 2012.

MONCAYO, A.; SILVEIRA, A.C. Current epidemiological trends for Chagas' disease in Latin America and future challenges in epidemiology, surveillance and health policy. Mem. Inst. Oswaldo Cruz, v. 104, p. 17-30, 2009. Supplement I.

NÓBREGA, A.A.; ARAÚJO, W.N.; VASCONCELOS. A.M.N. Mortality due to Chagas disease in Brazil according to a specific cause. Am. J. Trop. Med. Hyg., v. 91, n. 3, p. 528-533, 2014.

PEREIRA-BARRETO, A.C.; DEL CARLO, C.A.; CARDOSO, J.N. et al. Role of BNP levels on the prognosis of decompensated advanced heart failure. Arq. Bras. Cardiol., v. 100, n. 3, p. 281-287, 2013.

RASSI, A. JR.; RASSI, A.; MARIN-NETO. J.A. Chagas disease. Lancet, v. 375, n. 9723, p. 1388-1402, 2010.

SANGENIS, L.H.C.; SARAIVA, R.M.; GEORG, I. et al. Autochthonous transmission of Chagas disease in Rio de Janeiro State, Brazil: a clinical and eco-epidemiological study. BMC Infect. Dis., v. 15: p. 4, 2015.

SANGENIS, L.H.C.; SOUSA, A.S.; SILVA, G.M.S. et al. First report of acute Chagas disease by vector transmission in Rio de Janeiro State, Brazil. Rev. Inst. Med. Trop. São Paulo, v. 57, n. 4, p. 361-354, 2015.

SILVEIRA, A.C.; DIAS, J.C.P. 0 controle da transmissão vetorial. Rev. Soc. Bras. Med. Trop., v. 44, p. 5263, 2011. Suplemento II.

TOSO, A.M.; VIAL, F.U.; GALANTI, N. Transmisión de la enfermedad de Chagas por vía oral. Rev. Med. Chile, v 139, p. 258-266, 2011.

WORLD HEALTH ORGANIZATION. Research priorities for Chagas disease, human African trypanosomiasis and Leishmaniasis. Geneva: WHO, 2012. (Technical Report Series nº 975). 